\title{
5G New Radio Over Satellite Links: Synchronization Block Processing
}

\author{
Harri Saarnisaari \\ University of Oulu \\ Oulu, Finland \\ harri.saarnisaari@oulu.fi
}

\author{
Jean-Michel Houssin \\ Thales Alenia Space \\ Toulouse, France \\ Jean-Michel.Houssin@thalesaleniaspace.com
}

\author{
Thibault Deleu \\ Thales Alenia Space \\ Toulouse, France \\ Thibault.Deleu@thalesalenisaspace.com
}

\begin{abstract}
Harmonization of terrestrial standards such that they would support satellite elements would offer several benefits. This work has started at 3GPP relating to 5G new radio (NR). Downlink initial access is one of the topics that has to be evaluated in this respect among many others. This paper studies detection of the synchronization signals included in the synchronization (SS) block of the 5G NR signal in satellite channels that have large, up to $720 \mathrm{kHz}$, Doppler frequency shift at $30 \mathrm{GHz}$ carrier frequency. Furthermore, the reception of system information data in the SS block is considered. It is shown that using dedicated large frequency shift aware detectors it is possible to meet the 5G NR requirements in the detection part. However, the data part cannot be reliably received using one-shot reception at low signalto-noise values. Some alternative solutions are considered and evaluated though some of them require changes to the standard in order to be efficient.
\end{abstract}

\section{INTRODUCTION}

Satellite communication systems could carry a proprietary waveform or be based on standards like Digital Video Broadcasting (DVB). DVB-2SX is used for the forward direction and DVB-RCS2 for the return direction. However, DVB-RCS2 standard is often partially implemented in vendors solutions and does not enable full interoperability between vendors. It would be cost efficient and vendor friendly if a common standard could be applied. Since terrestrial communications systems have new though continuously developing standards about every ten years, those could be good starting points for satellite standards as well. However, terrestrial standards are not designed for satellite environments. Consequently, satellite system vendors need some efforts to transform terrestrial standards to fit their needs.

A better solution would be a standard that takes both terrestrial and satellite communication into account. In 2018, the satellite use case has been accepted to the $5 \mathrm{G}$ new radio (NR) road map at 3rd Generation Partnership Project (3GPP) and studies are ongoing to see how satellite use cases would affect the readily defined 5G NR standard [1]. SaT5G, an European Union $\mathrm{H} 2020$ programme research project [http://sat5gproject.eu/], is one opportunity where required changes are investigated and inputs for standardisation bodies created. This paper raises from these investigations. However, it would be

This work was performed in "Satellite and Terrestrial Network for 5G (SaT5G)" project in EU H2020 program, grand agreement No. 761413 and Academy of Finland 6Genesis Flagship (grant No. 318927). reformative if the next generation (beyond 5G) standard would include the satellite use case from the beginning.

In [2] several points of using 5G NR over satellite links are addressed mentioning the satellite Doppler frequency shift as one challenge. Terrestrial systems are typically planned for relative small carrier frequency offset (CFO). For example, at 3 $\mathrm{GHz}$ and $30 \mathrm{GHz}$ carrier frequencies with $1000 \mathrm{~km} / \mathrm{h}$ mobility (aircraft) the maximum CFO due to the Doppler frequency shift would be $\pm 2.8 \mathrm{kHz}$ and $\pm 28 \mathrm{kHz}$, respectively. However, in satellite systems corresponding CFO could be as large as $\pm 50 \mathrm{kHz}$ and $\pm 720 \mathrm{kHz}$ at $3 \mathrm{GHz}$ and $30 \mathrm{GHz}$, respectively, and their maximum change rates $600 \mathrm{~Hz} / \mathrm{s}$ and $8.2 \mathrm{kHz} / \mathrm{s}$ [1]. In the terrestrial case CFO uncertainty is within the subcarrier spacing (SCS) of 5G NR that could be $15 \mathrm{kHz}, 30 \mathrm{kHz}, 60$ $\mathrm{kHz}$ and $120 \mathrm{kHz}$ since only larger end SCS values are defined for carrier frequencies above $6 \mathrm{GHz}$, whereas in the satellite system the worst case CFO uncertainty could be significantly larger than the SCS.

Other concerns are the link budget and channel models. DVB-S2 uses modulation coding combinations that work down to $-3 \mathrm{~dB}$ signal-to-noise ratio (SNR) level per received symbol [3, Fig. 4]. These match with the 5G NR low order modulations with severe coding. Therefore, assuming that "5G" satellite systems have similar link budgets than DVBS2 system has, the expected link budget is not a problem. In the line-of-sight (LOS) case the satellite channel is typically frequency flat, i.e., merely a single path channel [1]. However, in direct user access when non-LOS situations may occur, multipath propagation causes frequency selectivity on the channel [1].

Particularly, in this paper the reception and processing of the synchronization signal (SS) block of 5G NR are studied. The SS block is send by cellular base stations and contains information that user devices use to synchronize with and identify the base station as well as data related to access process. Synchronization is based on the primary synchronization signal (PSS), identification to the secondary synchronization signal (SSS) and the aforementioned data contains so called master information block (MIB) and some other information [4].

The challenges are related to detection and data decoding with large CFO especially at the low signal-to-noise ratio (SNR) per resource element (RE) since receiver sensitivity 


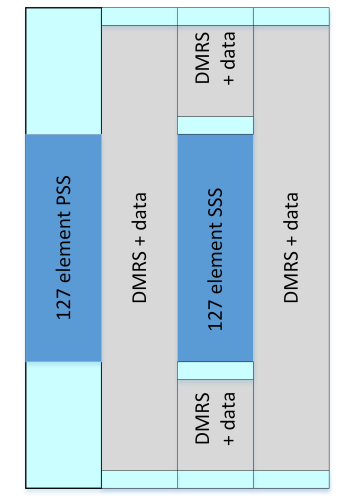

Fig. 1. The structure of the SS block.

is a requirement issue. The 5G NR standard says that PSS detection probability should be acceptable, close to or equal than $100 \%$ at $-6 \mathrm{~dB}$ with less than $1 \%$ false alarm rate $[5$, section 7.1.5] that is used as a low end baseline in this paper. Signal detection with large $\mathrm{CFO}$ is a familiar problem in satellite navigation systems and solutions exist as summarized, e.g., in [6]. By the authors' knowledge, 5G NR PSS detection with large CFO has not been considered elsewhere. Neither is SS block data demodulation and decoding considered in this environment. The aim of the paper is to provide insight could the SS block defined in the 5G NR standard be directly used in satellite access or not.

The next section describes the structure of the SS block including its signals, data and reference symbols for channel estimation as well as modulation and coding scheme selected for it in the 5G NR standard. Detection of the PSS signal with large CFO as well as the traditional approach for detection it are considered in section III followed by illustration of the problems it has and then by a discussion of a proper solution. This is followed by discussion about CFO estimation methods. Simulation results are shown in section VI including discussion about potential solutions to overcome the problems. The final section concludes the paper.

\section{SS BLOCK STRUCTURE}

The SS block [7, sec. 7.4.3] consist of 240 subcarriers on four consecutive 5G NR OFDM symbols. The first includes the 127 symbols PSS code formed by a spread spectrum code generator. The second and fourth symbol consist of reference symbols, known as demodulation reference signal (DMRS), at every fourth subcarrier and QPSK modulated data in others. The third symbol has a 127 symbol SSS code in the middle and 48 symbol subcarriers around it including DMRS at every fourth subcarrier. The general structure of the block is shown in Fig. 1. Altogether, there are 432 data subcarriers and 144 DMRS symbols. Since QPSK modulation is used data contains 864 coded bits. It should be mentioned that there are also other possibilities for PSS code and SCS that are alike what is in LTE, but those are not of interest herein.

The coding scheme is polar coding [8, section 7.1$]$. The MIB data is 24 bits [9] but with other data the information

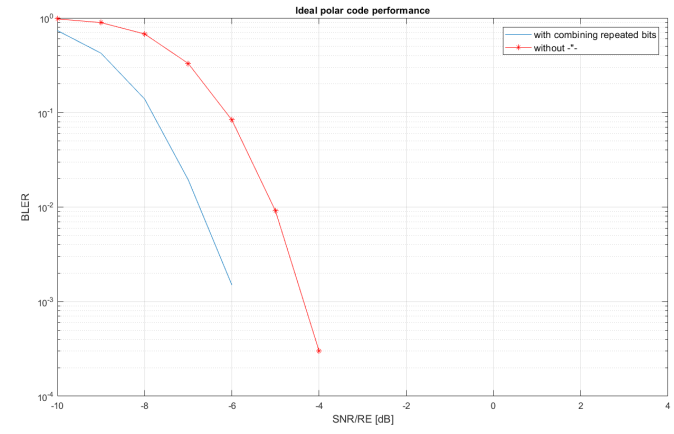

Fig. 2. The benchmark results for $\mathrm{PBCH}$ data.

content is about 30 bits depending on parameters [4] extended by 24 CRC bits. This totality is coded to 512 bits and then rate matched to 864 bits without forgetting to mention scrambling an interleaving functionalities [8]. The performed rate matching simply means that some bits are repeated. During decoding, the repeated bits can be utilized, e.g. by simple averaging or non-utilized, i.e. ignored, but the latter is definitely not an optimal way. The performance of the approach is sufficient in the ideal case even at low SNR as shown in Fig 2. This figure shows the performance of the described data and coding scheme in a known single path Gaussian channel without delay and CFO uncertainty. It can be seen that block error rate (BLER), especially using the average of repeated bits, is low, about $10^{-3}$ at $-6 \mathrm{~dB}$ satisfying the requirements whereas ignoring the repeated bit results $10^{-1}$ BLER. Herein, the polar coding scheme of the LTE5G toolbox provided by MATLAB was used as in the rest simulations. It includes coding, decoding and rate matching according the standard.

\section{PSS DETECTION}

In principle and in practice, detection of a 127 element direct sequence spread spectrum signal in an OFDM symbol is the problem. This is a well known problem and usual solution is the correlator or the matched filter receiver that have several implementation alternatives, either in a serial, parallel or one shot form. However, a large CFO is a problem like shown, e.g., in [10]. This effect is illustrated in the Fig. 3, where 30 $\mathrm{kHz}$ subcarrier spacing is used along with 5G NR PSS signal. It can be seen that detectability drops below the target level when CFO is $15 \mathrm{kHz}$ or larger and almost totally fails soon after this limit as the theory predicts. As a rule of thumb, the loss is $1 \mathrm{~dB}$ and $2 \mathrm{~dB}$ if $F T=0.25$ and $F T=0.35$, respectively, where $F$ is the $\mathrm{CFO}$ and $T$ the integration time (PSS code duration herein) [10]. Moreover, higher SNR means that more losses can be tolerated. The reason for this is that SNR at the detector output drops once CFO increases and at the certain moment the loss is too much for given input SNR. The results scale for the other SCS.

As a consequence, other means have to be used. In principle, such a one shot receiver contains parallel correlators 


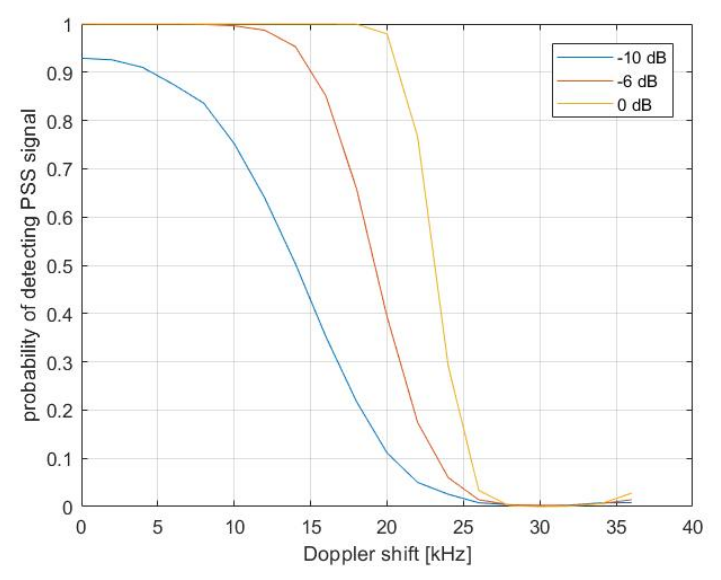

Fig. 3. PSS detection using the usual single channel detector.

or matched filters each matched to different candidate CFO. The separation of these candidates could be based on results in [10]. The rule of thumb is such that the separation should be such that in between the candidates the SNR loss (and detection sensitivity therefore) does not drop too much. If $F$ is the separation and $T$ the integration time (that varies depending on SCS), $F$ should be selected such that approximately $F T<2 / 3$. An approach to implement such a one shot detector is described in [6] and this approach is followed in this paper. In general, the larger the CFO uncertainty is, the more parallel correlators are needed. Therefore, the number of parallel detectors depends on operating frequency and satellite system type (GEO, MEO, LEO, HEO). The use of parallel correlators increases the receiver complexity, but that is the penalty that has to be paid. Another possibility is the seriel form receiver, but that increases time needed for initial synchronization, even remarkable.

In addition to offering detection of the PSS signal, the detector also provides time-of-arrival or delay estimate whose accuracy depends on the bandwidth, SNR, used oversampling rate and whose accuracy could be estimated using the CramerRao bound [11]. Furthermore, the detector provides a coarse initial CFO estimate.

\section{CFO ESTIMATION}

The initial CFO estimate is often not sufficient for data reception and it must be enhanced. There are various CFO estimators presented in the literature. A few simple ones are used in this paper. These are the cyclic prefix (CP) and DMRS symbol based estimators. The $\mathrm{CP}$ based estimator operates in the time domain and uses the $\mathrm{CP}$ and its counterpart in the OFDM symbol. Since the SS block contains four OFDM symbols, averaging could be over four OFDM symbols. A nice summary and theoretical performance of the $\mathrm{CP}$ based estimator are provided in [12]. The DMRS symbol based operates in the frequency domain and uses DMRS symbols at the second and fourth OFDM symbol of the SS block. Since there are several parallel DMRS symbols (at every fourth subcarrier) averaging in the frequency domain is possible. This is the well known data aided feed forward frequency estimator whose theoretical limit is also known [10].

\section{Simulator Model}

The SS block processing is modeled including the transmitter, single path channel, additive white Gaussian noise and receiver. As readily mentioned, the polar coding scheme from the LTE5G toolbox by MATLAB was used for the coding and decoding part. At the receiver the flow is as follows:

1) PSS detection. If not detected, the SS block is lost.

2) If the PSS signal was detected, time (sample moment) and initial CFO estimation are performed.

3) Downconversion by the initial CFO estimate.

4) SSS detection (simple correlator is sufficient) and enhanced CFO estimation.

5) Downconversion with the improved CFO estimate.

6) SS block data decoding preceded by channel estimation and equalization.

The channel estimator could use sole DMRS symbol but at low SNR this would be a rather low performing solution. Since there are possibilities that the system will be used in frequency fading channels full frequency domain averaging would not be the best solution either. Instead, 12 parallel (spread over 48 subcarriers) DMRS symbols are used in the averaging process and estimated channel tap is assumed to be valid over the corresponding band. The estimated channel tap is used to form a single tap zero forcing equalizer. The repeated bits are averaged before decoding.

\section{RESUltS}

One thousand Monte Carlo trials are executed for each SNR value. The SCS was set to $60 \mathrm{kHz}$. According the standard, the SS block is not send with this SCS. However, this SCS may be of interest for satellite systems and hence it was selected. Anyway, the results hold for other SCS though particular values would be different, multiplied or divided by relative SCS difference. For example, doubling the SCS would half the FT product since OFDM symbols would be shorter. This doubles the resistance against $\mathrm{CFO}$, but on the performance side it halves the integration time and worsens performance.

\section{A. Detection}

Detecting the PSS and SSS signals are of interest. The results are omitted since they were perfect, i.e., $100 \%$ detection success rate was achieved even at $-6 \mathrm{~dB} / \mathrm{RE}$. The used false alarm rate is $10^{-2}$ as required in the standard. The CFO and its change rate were randomly selected for each trial up to their maximum values $( \pm 720 \mathrm{kHz})$ and the receiver structure with parallel matched filters was used. Therefore, the design goal of the 5G NR standard can be achieved also with high CFO uncertainty if a proper detector is used. 


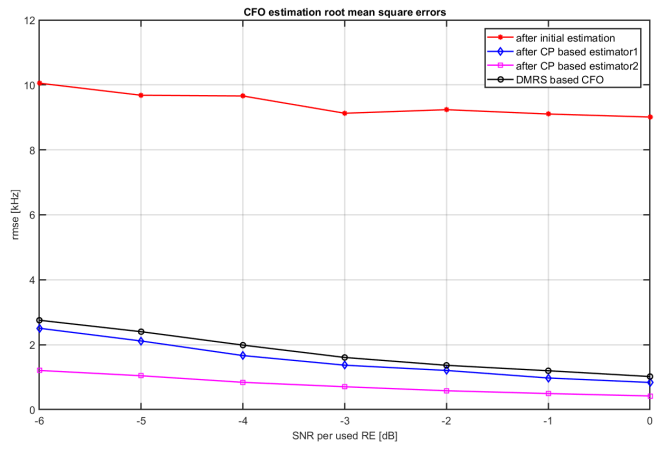

Fig. 4. CFO estimation errors for $30 \mathrm{GHz}$ case. On Y-axis rmse denotes root mean square error.

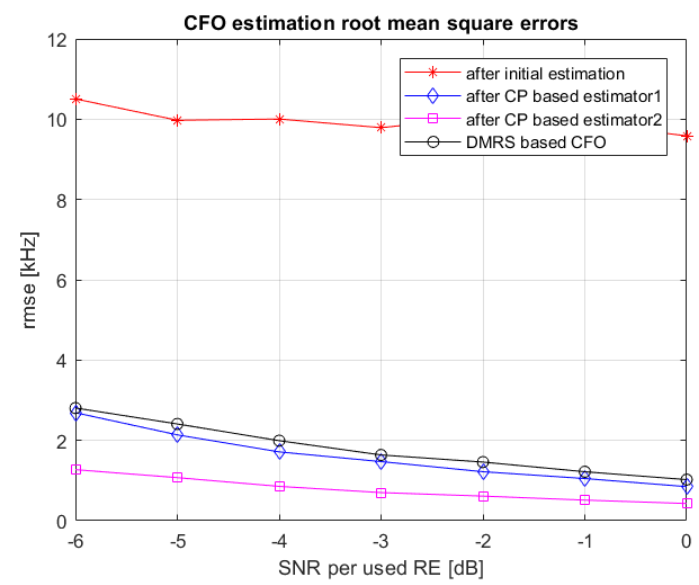

Fig. 5. CFO estimation errors for $3 \mathrm{GHz}$ case.

\section{B. CFO Estimation}

The CFO estimation accuracy using the three discussed estimators and the initial estimator are shown in Fig. 4. Estimator 1 refers to $\mathrm{CP}$ based estimator that uses sole OFDM symbol and estimator 2 to one that uses all four symbols. It can been that the estimators perform rather well also at low SNR values giving the data part a solid base. The obtained less than $3 \mathrm{kHz}$ error at low SNR is less than $5 \%$ of the subcarrier separation.

In addition to $30 \mathrm{GHz}$ carrier case also $3 \mathrm{GHz}$ carrier is simulated since therein the expected CFO range is much smaller. This is done to see if there is any difference. The results shown in Fig. 5 are similar than for the larger carrier frequency in Fig. 4 and, therefore, for larger CFO uncertainty. This is an expected observation since the theoretical and practical accuracy do not depend on the CFO uncertainty range. The purpose of the initial estimator is to bring $\mathrm{CFO}$ uncertainty within the pull-in range of the later CFO estimator.

\section{SS Block Data}

Based on the previous results the CFO estimator using all four symbols was selected for the data part. This means that the output of this particular estimator is used when downconverting the signal for data demodulation and decoding. However, before showing the results the ways to improve the process are discussed. In the simulations, 24 bits of data is used but difference to 30 bits is not significant. The estimated delay, CFO and channel are used. And only detected (i.e., PSS detected) SS blocks are processed and taken into account.

In order to overcome possible decoding issues that may occur at low SNR some countermeasures are possible. Those are discussed here. First, increasing the transmit power is not possible since the power at the satellite is limited. However, sending only SS block in those OFDM symbols would release all power to the SS block. How much depends on the total bandwidth and SCS. If SCS is $60 \mathrm{kHz}$, the SS block takes 14.4 $\mathrm{MHz}$ whereas for $120 \mathrm{kHz}$ SCS it takes $28.8 \mathrm{MHz}$. If the total bandwidth is $200 \mathrm{MHz}$, then the power of the SS block would increase approximately $11 \mathrm{~dB}$ and $8.5 \mathrm{~dB}$, respectively. However, this approach would somewhat decrease the throughput since some data subcarriers would not be used. Another mean is increasing the coding rate but that would require enlarging the SS block and, therefore, modifications to the standard.

The third possibility is decreasing the modulation order. This could be done using BPSK modulation instead of QPSK or by spreading the channel symbols. Spreading by factor two corresponds power increase provided by BPSK. The results in Fig. 6 show that the performance is not increased. This is so since the reduction of modulation order reduces the number of bits in the SS block and, consequently, the coding rate. It is well known that halving the code rate of the polar code decreases sensitivity by $3 \mathrm{~dB}$ [13]. Correspondingly, the benefits of having higher power per bit provided by the lower modulation order is counterbalanced by reduced coding gain. In order to gain from lower modulation order the SS block should be enlarged, i.e., the standard changed. Therefore, it has been proven here that the standard needs modifications before sensitivity of demodulating SS block data could be improved at low SNR values.

The fourth scheme, which does not require changes in the standard, is the use of higher DMRS power. However, since total power is fixed that would mean that the power of data symbols would be reduced. Let $P_{D M R S}$ and $P$ denote the power used for DMRS and data symbols, respectively. Then, the total power per four subcarriers is $P_{t o t}=P_{D M R S}+3 P$ and if $P_{D M R S}=a P, P=P_{t o t} /(3+a)$. If the share is equal $(a=1)$, each subcarrier has $25 \%$ of the total power. If the $P_{D M R S}$ is $3 \mathrm{~dB}(a=2)$ or $10 \mathrm{~dB}(a=10)$ higher, then data subcarriers get $1 / 5$ and $1 / 13$ of the total power, or $20 \%$ and $8 \%$, respectively. The former means $1 \mathrm{~dB}$ and the latter $5 \mathrm{~dB}$ reduction in data symbols' power. The simulation results shown in 6 are for given SNR/RE per data symbol (subcarrier). Since the total power is fixed, a fair comparison with other results means that $1 \mathrm{~dB}$ lower SNR/RE values should be looked at in the case of $3 \mathrm{~dB}$ larger DMRS power, i.e., that $-5 \mathrm{~dB}$ point is actually $-6 \mathrm{~dB}$ point of data symbols. For $3 \mathrm{~dB}$ higher DMRS power this means that performance is not $10^{-3}$ at $-5 \mathrm{~dB}$ but $2 \times 10^{-2}$ (read from $\left.-6 \mathrm{~dB}\right)$. As 


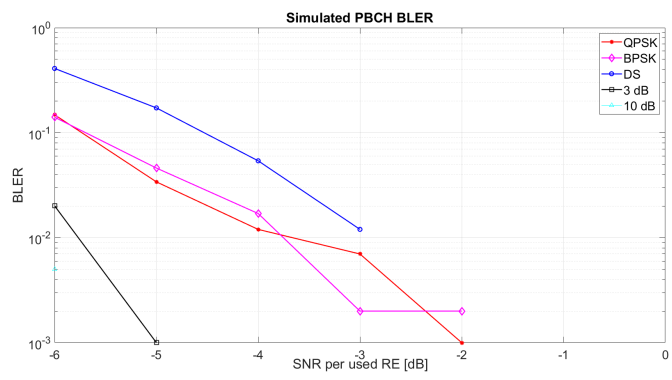

Fig. 6. Simulated BLER for the SS block data when a) BPSK and b) QPSK are used and QPSK with $3 \mathrm{~dB}$ or $10 \mathrm{~dB}$ higher DMRS power or with four times spreading (DS).

a conslusion, the results are better with the increased DMRS power and with $10 \mathrm{~dB}$ higher DMRS power, packet errors did not occur at the given SNR range. Consequently, since the performance improves with increasing DMRS power, the result mean that channel estimation quality at low SNR plays a crucial role if ideal BLER results are hunted.

Finally, it is reminded that it is possible to use consecutive SS blocks to demodulate the data reliably. The blocks are send frequently, every $80 \mathrm{~ms}$ or so, such that this does not take too much extra time in initial access process. This does not require changes in the standard and has been mentioned as a processing alternative [5, section 7.1.5].

\section{CONCLUSIONS}

The paper has shown that it is possible to meet $5 \mathrm{G}$ NR PSS detection requirements (at $-6 \mathrm{~dB} / \mathrm{RE}$ ) also on satellite channels where CFO uncertainty range is much larger than in the terrestrial case for which $5 \mathrm{G}$ NR was designed. However, a proper large $\mathrm{CFO}$ aware detector must be used that provides an initial $\mathrm{CFO}$ estimate. After the sufficient initial CFO estimation, usual CFO estimators can be used for improving CFO estimation as was shown in this paper. These bring CFO error below an acceptable level. Indeed, their performance is similar than in the terrestrial case. At low SNR, between 6 and $-3 \mathrm{~dB} / \mathrm{RE}$ (per resource element), the decoding of the system information included into the SS block is challenging and block error rate is up to $10^{-1}$ (see Fig. 6) but this holds also in the terrestrial case. Some alternatives to overcome the problem were discussed of which some require changes to the standard to be effective. Repeated reception of this data (that is unaltered) is one option mentioned in the standard.

As a conclusion, it can be said that the 5G NR SS block design is adequate also for satellite channels assuming that SNR is larger than $-6 \mathrm{~dB} / \mathrm{RE}$ if a proper, large $\mathrm{CFO}$ aware PSS detector is used. After initial CFO estimation provided by this detector the processing is similar than in the terrestrial case.

All the aspects of the satellite transmission chain were not studied. Therefore, future works could include investigations on multipath channels [1] met in the direct end user access. Furthermore, effects of satellite amplifier nonlinearity should be evaluated. This means that peak-to-average-power ratio
(PAPR) reduction methods used at the transmitter as well nonlinear power amplifier at (pent-pipe) satellites should be modeled.

Similar processing is done also in uplink direction. In the initial access process user device sends random access signal (PRACH) to the base station that has to detect it, in a satellite channel with unknown potentially large Doppler frequency shift. Therefore, the processing should be similar than discussed herein. Indeed, it is expected that detection results shown here hold also for PRACH signal that is very similar than PSS. Indeed, PRACH could be a longer signal if a proper PRACH format is selected (by $\mathrm{gNB}$ ) meaning that it could be detected even at lower SNR values.

\section{REFERENCES}

[1] 3rd Generation Partnership Project; Technical Specification Group Radio Access Network, "Study on new radio (NR) to support non terrestrial networks (release 15)," 2018, 3GPP TR 38.811 V15.0.0 (2018-06).

[2] O. Kohdeli, A. Guidotti, and A. Vanelli-Coralli, "Integration of satellites in $5 \mathrm{~g}$ through leo constellations," in Globecom, 2017.

[3] ETSI, "Digital video broadcasting (DVB); implementation guidelines for the second generation system for broadcasting, interactive services, news gathering and other broadband satellite applications; part 1: DVB-S2," 2015, ETSI TR 102 376-1 V1.2.1 (2015-11).

[4] E. Dahlman, S. Parkval, and J. Sköld, 5G NR The Next Generation Wireless Access Technology. Academic Press, 2018.

[5] 3rd Generation Partnership Project; Technical Specification Group Radio Access Network, "Study on new radio access technology physical layer aspects (release 14)," 2017, 3GPP TR 38.802 V14.2.0 (2017-09).

[6] H. Saarnisaari and E. Karami, "Frequency domain block filtering GNSS receivers," in Proceedings of the IEEE Position Location and Navigation Symposium, Monterey, CA, May 2008.

[7] 3rd Generation Partnership Project; Technical Specification Group Radio Access Network, "NR; physical channels and modulation (release 15)," 2018, 3GPP TS 38.211 V15.3.0 (2018-09).

[8] - "NR; multiplexing and channel coding (release 15)," 2018, 3GPP TS 38.212 V15.2.0 (2018-06).

[9] sharetechnote.com, "5G/NR - SS Block," [0nline] http://www. sharetechnote.com/html/5G/5G_SS_Block.html, [Accessed: Jan- 2019].

[10] O. C. Maus, F. Classen, and H. Meyr, "Carrier frequency recovery for a fully digital direct-sequence spread-spectrum receiver: A comparison," in Proceedings of the IEEE Vehicular Technology Conference, 1993, pp. 392-395.

[11] H. Saarnisaari, "Some design aspects of mobile local positioning systems," in Proceedings of the IEEE Position Location and Navigation Symposium, Monterey, CA, USA, 2004.

[12] T.-C. Lin and S.-M. Phoong, "A new cyclic-prefix based algorithm for blind CFO estimation in OFDM systems," IEEE TRANSACTIONS ON WIRELESS COMMUNICATIONS, vol. 15, no. 6, pp. 3395-4008, June 2016.

[13] A. Sharma and M. Salim, "Polar code: The channel code contender for 5G scenarios," in International Conference on Computer, Communications and Electronics (Comptelix), 2017. 